\title{
COGNITIVE BIASES AND CULTURAL EVOLUTION IN THE EMERGENCE OF SPACE-TIME MAPPINGS IN LANGUAGE
}

\author{
TESSA VERHOEF ${ }^{* 1,2}$, ESTHER WALKER $^{3}$, and TYLER MARGHETIS ${ }^{4}$ \\ *Corresponding Author: tverhoef@ucsd.edu \\ ${ }^{1}$ Leiden Institute of Advanced Computer Science, Leiden University, Leiden, The \\ Netherlands \\ ${ }^{2}$ Center for Research in Language, University of California, San Diego, La Jolla, USA \\ ${ }^{3}$ Cognitive Science, University of California, San Diego, La Jolla, USA \\ ${ }^{4}$ Psychological and Brain Sciences, Indiana University, Bloomington, USA
}

Humans spatialize time. This occurs in artifacts like timelines, in gesture, and critically — in conventional language ("look FORWARD to the future"). This phenomenon, however, exhibits considerable cross-linguistic variability. Explaining the origins of space-time mappings in language, therefore, will require considering both cultural processes and individual biases (Núñez \& Cooperrider, 2013). Here we present two laboratory experiments on the interplay of these mechanisms in the emergence of space-time mappings.

In our first experiment, pairs of English-speaking participants (i.e., dyads) played a guessing game (cf. Garrod et al., 2007; Healey et al., 2007; etc.) about temporal concepts, using a novel, spatial signaling device: a vertical bar with a moving bubble controlled through a touch screen (Fig. 1a). After repeated interaction and social coordination, dyads developed shared signals that used space to express aspects

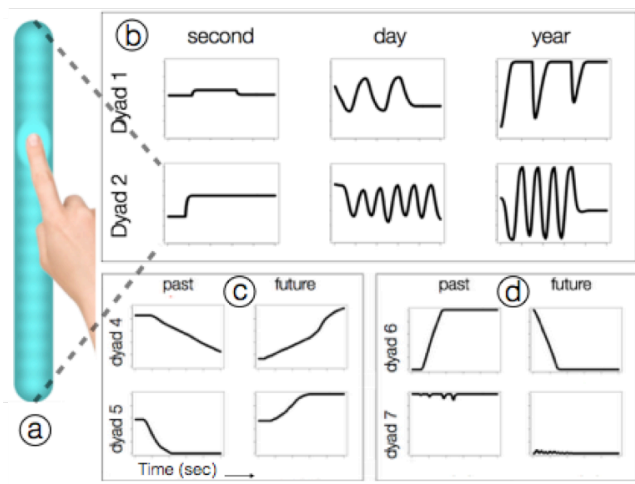

Figure 1: (a) Signaling device, (b) Consistent use of spatial length to communicate relative duration, (c) and (d) Contrasting mappings for past/future. 
of time. Some of these conventions were nearly universal. For instance, every dyad used greater spatial lengths to indicate greater temporal durations (Fig. 1b). Conventions using vertical location to differentiate past from future also recurred across dyads, but they differed in their precise mapping (e.g., up = past or up = future, (Fig. 1c, d)). This mix of shared and idiosyncratic conventions suggests the involvement of both shared biases and cultural processes. While all dyads developed space-time mappings, which facilitated communication, these were never sufficiently elaborated or systematic to achieve perfect communication.

Our second experiment tested how transmission would further shape the signalling systems that emerged in the guessing game from Experiment 1. We hypothesized that iterated transmission across generations of interacting users might prompt the evolution of more regular and stable systems (Tamariz

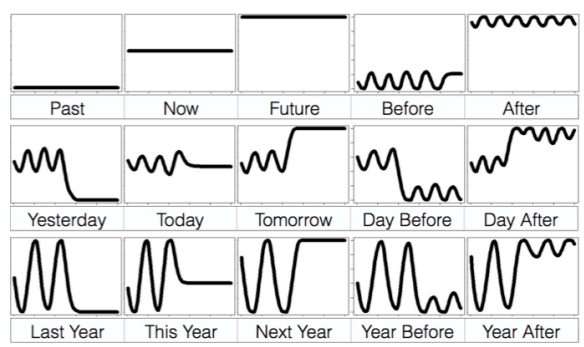

Figure 2: A system of signal-meaning mappings et al, 2012). The signals that were developed by one dyad were used as initial training for the next dyad, before they started interacting. We tested six chains of eight dyads. After repeated transmission, some dyads achieved near-perfect communicative success, as a result of the gradual appearance of more fully systematized space-time mappings. Figure 2 illustrates an emerged system, which evolved highly systematic mappings and compositionality.

These laboratory experiments illustrate how space-time mappings in language can evolve from interacting mechanisms involving individual biases, social coordination and iterated transmission. The interplay between these mechanisms may explain the cross-linguistic commonalities as well as the variety found in space-time mappings.

\section{References}

Garrod, S., Fay, N., Lee, J., Oberlander, J. \& MacLeod, T. (2007). Foundations of representation: Where might graphical symbol systems come from? Cognitive Science, 31(6): 961-987.

Healey, P. G. T., Swoboda N., Umata I. \& King J. (2007). Graphical language games: interactional constraints on representational form. Cognitive Science, 31: 285-309. 
Núñez, R. \& Cooperrider, K., (2013). The Tangle of Space and Time in Human Cognition. Trends in Cognitive Sciences, 17(5): 220-229.

Tamariz, M., Cornish, H., Roberts, S. \& Kirby, S. (2012) The effect of generation turnover and interlocutor negotiation on linguistic structure. In Proceedings of EVOLANG9. World Scientific, (pp. 555-556). 